\title{
Diminished activity of tartrate resistant acid phosphatase in alveolar macrophages from patients with active sarcoidosis
}

\author{
J BARTH, HANS KREIPE, JOACHIM KIEMLE-KALLEE, HEINZ J RADZUN, \\ MOHAMMAD R PAR WARESCH, WOLFGANG PETERMANN
}

From the Departments of Medicine and Pathology, University of Kiel, Kiel, Federal Republic of Germany

ABSTRACT Alveolar macrophages differ from their percursors in blood, monocytes, by expressing strong activity of the tartrate resistant variant of acid phosphatase (TAcP). A study was carried out to analyse the expression of this enzyme cytochemical marker by alveolar macrophages from bronchoalveolar lavage cells from 34 patients with sarcoidosis and 12 control subjects. Alveolar macrophages from control subjects displayed a strong and homogeneous staining pattern and only $0.1 \%$ of cells were negative after staining. Macrophages from patients with sarcoidosis showed reduced TAcP activity and up to $7 \%$ of the cells were negative. The percentage of TAcP negative macrophages was correlated with the percentage of lymphocytes and with the ratio of CD4 to CD8 lymphocytes among cells recovered by bronchoalveolar lavage. The reduced TAcP activity in alveolar macophages from patients with sarcoidosis may be due to an increased recruitment of immature precursors from blood.

\section{Introduction}

Alveolar macrophages are derived from blood monocytes.' The maturation of alveolar macrophages from their precursors in blood is characterised by the aquisition of varied phenotypic and functional properties. ${ }^{2}$ Among these tartrate resistant acid phosphatase (TAcP) provides an easily detectable enzyme cytochemical marker for alveolar macrophages. ${ }^{3}$

The alveolitis associated with pulmonary sarcoidosis is characterised by changes in the number, surface phenotype, and activity of both alveolar macrophages and $\mathrm{T}$ lymphocytes. ${ }^{45}$ Although various procedures have been proposed to evaluate the lymphocytes of the alveolitis of sarcoidosis, ${ }^{5}$ techniques for recording the macrophage component of the alveolitis are not well established. Hance et al have described changes in the surface phenotype of alveolar macrophages in pulmonary sarcoidosis, which probably resulted, at least in part, from an increased recruitment of young cells to the lung. ${ }^{6}$ This study investigated the expression of TAcP activity, a cyto-

Address for reprint requests: Dr Jürgen Barth, I Medizinische Universitätsklinik, Schnittenhelmstr 12, D-2300 Kiel, Federal Republic of Germany.

Accepted 9 August 1988 chemical marker for mature alveolar macrophages, by alveolar macrophages from patients with pulmonary sarcoidosis. Our results show that alveolar macrophages in sarcoidosis have diminished TAcP activity, which may be due to an increased influx of alveolar macrophage precursors into the alveoli.

\section{Methods}

We investigated 34 patients with pulmonary sarcoidosis (mean age 37.8 (SD 9.8) years). The diagnosis of sarcoidosis was confirmed by clinical, radiographic, and histological features in all cases. To determine whether the proportion of TAcP positive alveolar macrophages correlated with the number or type of lymphocytes recovered by lavage or with the pattern observed on the chest radiograph, the patients were grouped in the following ways: (1) On the basis of the lymphocyte count in the bronchoalveolar lavage fluid-22 patients had a lymphocyte count of less than $30 \%$ and 12 a lymphocyte count of $30 \%$ or more. (2) On the basis of the T4:T8 ratio- 21 patients had a ratio of less than 5 and 13 a ratio 5 or more. (3) On the basis of the findings on the chest radiograph-21 patients had stage I (hilar adenopathy without pulmonary disease) and 13 stage II (hilar adenopathy with pulmonary disease) radiographic changes. 
Twelve subjects (mean age 51 (SD 14) years) served as the control group. In the eight with a small peripheral bronchial carcinoma the unaffected lung was lavaged. Four patients were investigated to exclude pulmonary manifestations of collagen disease. None of the control subjects had abnormal differential counts of the cells recovered by lavage.

Blood monocytes from eight healthy volunteers (mean age 24 (SD 3) years) and from 12 patients with sarcoidosis (mean age $34(5)$ years) were separated by density gradient centrifugation followed by glass adherence. Cells were harvested by gentle scraping with a rubber policeman.' Bronchoalveolar lavage of the right middle lobe or lingula was performed.' Pappenheim staining and immunocytochemical staining of cytospin preparations from lavage cells served to determine the lymphocyte count and the percentage of T lymphocyte subpopulations respectively. 'Acid phosphatase activity was visualied by using naphtolAS-BI-phosphate (Sigma, Munich) as substrate and hexazotised pararosaniline as coupler according to the method of Barka and Anderson. ${ }^{8}$ TAcP staining was performed by adding 0.05 M tartrate (Merck, Darmstadt) to the incubation medium for visualisation of acid phosphatase.

Enumeration of lymphocytes, macrophages, and lymphocyte subpopulations was performed by counting 200 cells. To determine the number of TAcP negative alveolar macrophages 1000 cells were coun- ted. To ensure that the TAcP negative cells counted were really alveolar macrophages, only those cells with? a typical macrophage morphology-that is, showing phagocytosed particles-were counted as TAcP $\overline{\frac{0}{\bar{m}}}$ negative alveolar macrophages.

\section{Results}

Blood monocytes from normal donors and patients with sarcoidosis did not show TAcP activity. By contrast, alveolar macrophages from the contro氶 group showed a diffuse and intense TAcP stainingo pattern (fig la). Very few alveolar macrophages from control subjects were totally TAcP negative $(0.13 \%$ is fig 2).

Alveolar macrophages from the patients with saro coidosis, however, showed reduced TAcP activity onthe basis of staining. The staining pattern was charac $z$ terised by a spotty and inhomogeneous distribution and the proportion of total TAcP negative alveolas macrophages was considerably greater (up to $7 \%$ than in the control group.

In the subgroup analysis of patients with sarcoidosi£ there were more TAcP negative alveolar macrophages in patients with a high percentage of lymphocytes iß the lavage fluid, in those with an increased T4:T8 ratios and in those with stage II radiographic appearances (fig 2). The greatest number of TAcP negative alveolao

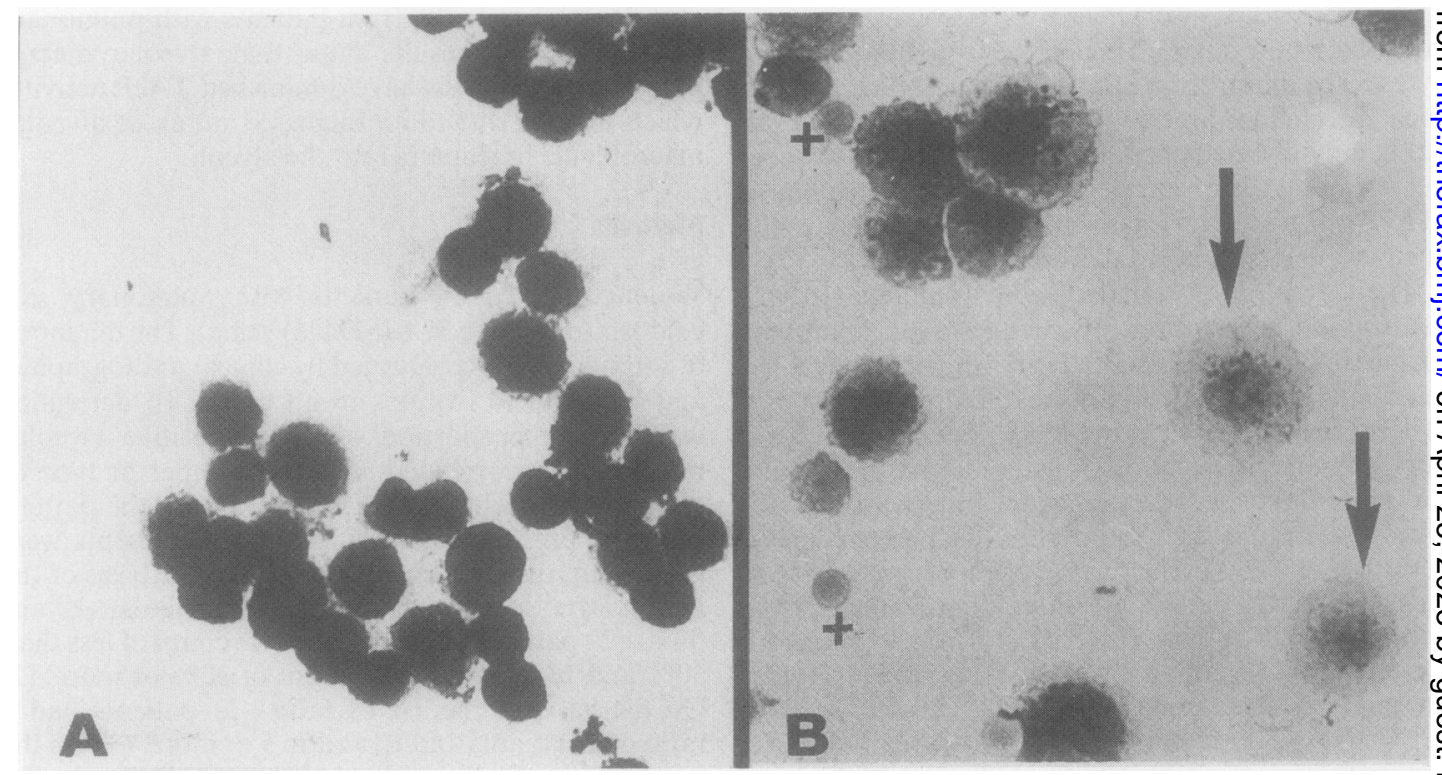

Fig 1 Cytospin preparations of alveolar macrophages stained for tartrate resistant acid phosphatase (TAcP). (A) Strong and uniform TAcP staining for alveolar macrophages from a patient with a small peripheral bronchial carcinoma. TAcP negative alveolar macrophages are not detectable. (B) Diminished TAcP staining in alveolar macrophages from a patient with active pulmonary sarcoidosis. Arrows indicate TAcP negative alveolar macrophages and crosses lymphocytes. 


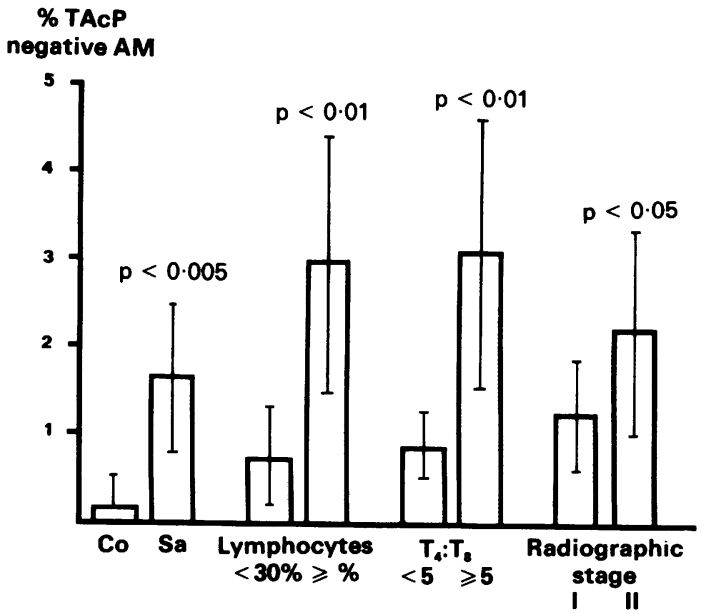

Fig 2 Percentages of tartrate resistant acid phosphatase (TAcP) negative alveolar macrophages $(A M)$ in control subjects and the various groups of patients with sarcoidosis (means with SD). Co-controls; Sa-patients with sarcoidosis.

macrophages occurred in patients with a T4:T8 ratio of 5 or more (fig 2).

Regression analysis showed a positive correlation between the number of lavage fluid lymphocytes and the number of TAcP negative alveolar macrophages $(r=0.758, p<0.005)$. A less striking but still significant correlation was found between the $T 4$ : $T 8$ ratio and the number of TAcP negative alveolar macrophages in patients with sarcoidosis $(r=0.671$, $\mathrm{p}<0.005)$.

\section{Discussion}

Several lines of evidence indicate that the alveolitis associated with pulmonary sarcoidosis leads to an increased recruitment of monocytes into the lung. Alveolar macrophages in sarcoidosis differ from normal resident macrophages by having a more "immature" surface phenotype, resembling that of blood monocytes. ${ }^{6-9}$ The evidence that alveolar macrophages in sarcoidosis, unlike other macrophage populations, share with blood monocytes the capacity to release a type IV collagenase supports the idea that monocytes actively migrate from the blood to the lung in this disease. ${ }^{10}$ Moreover, more alveolar macrophages in sarcoidosis retain the ability to proliferate, thus indicating a lower stage of maturation. ${ }^{11}$

Our finding of reduced TAcP expression in alveolar macrophages from patients with sarcoidosis (fig 1) accords well with the view that the alveolar macrophages in the alveoli in this disease are immature. Blood monocytes from control subjects and from patients with sarcoidosis showed no TAcP activity, whereas alveolar macrophages from control subjects displayed a strong and homogeneous expression of this cytochemical marker. In addition to the reduced TAcP activity observed in almost all alveolar macrophages in the patients with sarcoidosis, there was an increased number of completely TAcP negative alveolar macrophages. When numbers of TAcP negative macrophages were related to indices that presumably mirror disease activity, such as the percentage of lymphocytes among lavage cells and the CD4:CD8 ratio, significantly more negative macrophages were found in patients with more than $30 \%$ of lymphocytes and a CD4:CD8 ratio of 5 or more.

Reduction or absence of TAcP activity was observed in some alveolar macrophages with an apparently mature morphology (fig 1). As macrophages are known to secrete lysosomal enzymes actively in response to an inflammatory challenge, ${ }^{12}$ active secretion of these enzymes is an additional mechanism that might account for the reduced TAcP activity in patients with sarcoidosis. The TAcP content of bronchoalveolar lavage fluid must be analysed to test this hypothesis. Further studies are also necessary to evaluate the prognostic relevance of TAcP staining in patients with sarcoidosis, as this may represent an easily detectable marker of disease activity.

\section{References}

1 Radzun HJ, Parwaresch MR, Kreipe H. Monocytic origin of human alveolar macrophages. $J$ Histochem Cytochem 1983;31:318-24.

2 Blussé Van Onu Alblas A, Van Furth R. Origin, kinetics and characteristics of pulmonary macrophages in the normal steady state. J Exp Med 1979;149:1504-18.

3 Radzun HJ, Kreipe H, Parwaresch MR. Tartrate-resistant acid phosphatase as a differentiation marker for the human mononuclear phagocyte system. Hematol Oncol 1983;1:321-7.

4 Thomas PD, Hunninghake GW. Current concepts on the pathogenesis of sarcoidosis. Am Rev Respir Dis 1987;135:747-60.

5 Hunninghake GW, Crystal RG. Pulmonary sarcoidosis: a disorder mediated by excess helper T-lymphocyte activity at site of disease activity. $N$ Engl $J$ Med 1981;305:429-34.

6 Hance AJ, Douches S, Winchester AJ, Ferrans VJ, Crystal RG. Characterization of mononuclear phagocyte subpopulations in the human lung by using monoclonal antibodies: changes in alveolar macrophage phenotype associated with pulmonary sarcoidosis. J Immunol 1985;134:284-92.

7 Barth J, Entzian P, Petermann W. Increased release of free oxygen radicals by phagocytosing and nonphagocytosing cells from patients with active pulmonary sarcoidosis as revealed by luminol-dependent chemiluminescence. Klin Wochenschr 1988;66:292-7. 
8 Barka T, Anderson PJ. Histochemical methods for acid phosphatase using hexazonium pararosaniline as coupler. J Histochem Cytochem 1962;10:741-53.

9 Petermann W, Barth J, Entzian P, Kreipe H, Radzun HJ, Parwaresch MR. Phenotypic differentiation of alveolar macrophages from normal subjects and sarcoidosis patients using new monocyte/macrophage specific monoclonal antibodies of the $\mathrm{Ki}-\mathrm{M}$ series [abstract]. Am Rev Respir Dis 1987;135:A29.

10 Garbisa S, Talin M, Daga-Gordini D, et al. Type IV collagenotic activities by human macrophages: purification and characterization of a specific $68 \mathrm{KD}$ protease. $J$ Biol Chem 1986;261:2369-75.

11 Bitterman PB, Saltzman LE, Adelberg S, Ferrans VJ, Crystal RG. Alveolar macrophage replication. One mechanism for the expansion of the mononuclear phagocyte population in the chronically inflamed lung. J Clin Invest 1984;74:460-9.

12 Nathan CF, Murray HW, Cohn ZA. The macrophage as an effector cell. $N$ Engl J Med 1980;303:622-6. 